\title{
FREE FIELDS IN MALCEV-NEUMANN SERIES RINGS
}

\author{
VITOR O. FERREIRA, ÉRICA Z. FORNAROLI, AND JAVIER SÁNCHEZ
}

\begin{abstract}
It is shown that the skew field of Malcev-Neumann series of an ordered group frequently contains a free field of countable rank, i.e. the universal field of fractions of a free associative algebra of countable rank. This is an application of a criterion on embeddability of free fields on skew fields which are complete with respect to a valuation function, following K. Chiba. Other applications to skew Laurent series rings are discussed. Finally, embeddability questions on free fields of uncountable rank in Malcev-Neumann series rings are also considered.
\end{abstract}

\section{INTRODUCTION}

The existence of free objects in skew fields has attracted considerable attention since Lichtman 16 conjectured that the multiplicative group of a noncommutative skew field contains noncyclic free subgroups. A related problem, regarding free subalgebras inside skew fields, has also been the subject of investigation although somewhat less intensely. And free group algebras have been conjectured to exist in finitely generated skew fields which are infinite-dimensional over their centres. We refer to the survey article [10] and the references therein.

More recently, K. Chiba [3] has considered complete skew fields with respect to a valuation function and proved that they contain free fields provided they are infinite-dimensional over their centres. Moreover, G. Elek [8] has considered free subfields of skew fields in the context of amenability.

In this paper, we present some improvements on Chiba's results which yield conditions under which skew fields of Malcev-Neumann series of ordered groups are shown to contain free fields of countable rank. These valuation methods are later also applied to skew Laurent series rings.

A remark of A. Lichtman on the nonexistence of free fields in the field of fractions of group algebras of torsion free polycyclic-by-finite groups is presented in Section 4 , showing that the free algebras that are known to exist in these skew fields do not generate free fields.

Date: 16 August 2011.

2000 Mathematics Subject Classification. 16K40, 16W60, 16S10.

Key words and phrases. Division rings, free fields, valuations, Malcev-Neumann series.

The first author is the corresponding author and was partially supported by CNPq, Brazil (Grant 308163/2007-9).

The second author was supported by CNPq, Brazil (Grant 141505/2005-2).

The third author was partially supported by FAPESP, Brazil (Proc. 2009/50886-0), by the DGI and the European Regional Development Fund, jointly, through Project MTM200806201C0201, and by the Comissionat per Universitats i Recerca of the Generalitat de Catalunya, Project 2009 SGR 1389. 
A final section is devoted to the generalization of the valuation methods used earlier in order to guarantee the presence of free fields of uncountable rank in some Malcev-Neumann series rings.

\section{Definitions AND NOtATion}

In what follows the terms "division ring" and "skew field" will be used interchangeably. Occasionally, we shall omit the adjective "skew". A skew field which is commutative will always be called a "commutative field".

Given a skew field $D$, a subfield $K$ of $D$ and a set $X$, the free $D_{K}$-ring on $X$ is defined to be the ring $D_{K}\langle X\rangle$ generated by $X$ over $D$ satisfying the relations $a x=x a$ for all $a \in K$ and $x \in X$. It is well known that $D_{K}\langle X\rangle$ is a fir and, thus, has a universal field of fractions $D_{K}(\langle X\rangle)$, called the free $D_{K}$-field on $X$. (We refer to [5] for the theory of firs and their universal fields of fractions.) The free $D_{D}$-ring on $X$ will be called the free $D$-ring on $X$ and will be denoted by $D\langle X\rangle$. Its universal field of fractions, the free $D$-field, will be denoted by $D(\langle X\rangle)$.

We shall be looking at valuation functions on skew fields. Let $R$ be a ring and let $(G,<)$ be a (not necessarily abelian) ordered group with operation denoted additively. A symbol $\infty$ is adjoined to $G$ and in $G_{\infty}=G \cup\{\infty\}$ the operation and order are extended in such a way that $x+\infty=\infty+x=\infty+\infty=\infty$ and $x<\infty$, for all $x \in G$. By a valuation on $R$ with values in $G$ one understands a map $\nu: R \rightarrow G_{\infty}$ satisfying

(i) $\nu(a)=\infty$ if and only if $a=0$,

(ii) $\nu(a b)=\nu(a)+\nu(b)$, for all $a, b \in R$,

(iii) $\nu(a+b) \geq \min \{\nu(a), \nu(b)\}$, for all $a, b \in R$.

The valuation $\nu$ is said to be trivial if $\nu(a)=0$, for all $a \in R, a \neq 0$. When $R$ is a skew field and $R^{\times}$denotes the set of all nonzero elements of $R, \nu\left(R^{\times}\right)$is a subgroup of $G$, called the value group of $\nu$. A valuation on a skew field with value group order-isomorphic to the ordered group of the integer numbers is said to be discrete.

We shall be mainly concerned with valuations on skew fields with values in $\mathbb{R}$, the ordered additive group of the real numbers with its usual order. If $D$ is a skew field and $\nu: D \rightarrow \mathbb{R}_{\infty}$ is a valuation, we can define a metric $d$ on $D$ by choosing a real constant $c \in(0,1)$ and letting $d(a, b)=c^{\nu(a-b)}$, for all $a, b \in D$. The topology so defined on $D$ is independent of the choice of the constant $c$, and the completion $\widehat{D}$ of the metric space $D$ is again a skew field with a valuation $\hat{\nu}: \widehat{D} \rightarrow \mathbb{R}_{\infty}$ such that $\widehat{D}$ and $\hat{\nu}$ are extensions of $D$ and $\nu$, respectively. We shall refer to $\widehat{D}$ as the completion of $D$ with respect to the valuation $\nu$.

An example is the following. Let $R$ be a ring with a central regular element $t$ such that $\bigcap t^{n} R=0$ and $R / t R$ is a domain. If we define, for each $a \in R$, $\nu(a)=\sup \left\{n: a \in t^{n} R\right\}$ then $\nu$ is a valuation on $R$, called the $t$-adic valuation. In particular, if $R$ is a domain and if $R[z]$ is the polynomial ring over $R$ on the indeterminate $z, R[z]$ has a $z$-adic valuation. If, moreover, $R$ is a division ring, then $R[z]$ is an Ore domain with field of fractions $R(z)$, the field of rational functions on $z$. The $z$-adic valuation on $R[z]$ extends to a discrete valuation $\nu$ on $R(z)$ and the completion of $R(z)$ with respect to $\nu$ is just the field of Laurent series $R((z))$.

The following further notions regarding ordered groups will be needed in the sequel. Details can be found in [2] or [9, for example. 
Given an ordered group $(G,<)$, a subgroup $H$ of $G$ is said to be convex if for all $x, z \in H$ and $y \in G$, the inequalities $x \leq y \leq z$ imply that $y \in H$. If a convex subgroup $N$ is normal, then $G / N$ has a natural order inherited from that on $G$, and the convex subgroups of $G / N$ are in one-to-one correspondence with those of $G$ which contain $N$.

The ordered group $(G,<)$ is said to be archimedean if $\{0\}$ and $G$ are the only convex subgroups of $G$. An archimedean group is known to be order isomorphic to a subgroup of the additive group $\mathbb{R}$ of the real numbers with its natural order.

The set of convex subgroups of an ordered group $(G,<)$ is totally ordered with respect to inclusion, and we say that a pair $(N, H)$ of convex subgroups of $G$ is a convex jump if $N \varsubsetneqq H$ and there are no convex subgroups lying properly between $N$ and $H$. Given a convex jump $(N, H), N$ is known to be normal in $H$ and, therefore, $H / N$ is order isomorphic to a nontrivial subgroup of $\mathbb{R}$.

Finally, we recall that given a skew field $K$ and an ordered group $(G,<)$, the Malcev-Neumann series ring $K((G,<))$ is defined to be the set of all formal series $f=\sum_{x \in G} x a_{x}$, with $a_{x} \in K$, whose support $\operatorname{supp}(f)=\left\{x \in G: a_{x} \neq 0\right\}$ is a well-ordered subset of $G$. The operations of $K$ and $G$, together with the relations $a x=x a$, for all $a \in K$ and $x \in G$ induce a multiplication in $K((G,<))$, with respect to which $K((G,<))$ is a field. Clearly, $K((G,<))$ contains the group ring $K[G]$ of $G$ over $K$. We denote by $K(G)$ the subfield of $K((G,<))$ generated by $K[G]$. In Section 3, we shall encounter a more general construction.

\section{Free Fields in VALUED DiVISION RINGS}

We start by remarking that Chiba's main result in [3] holds in a more general setting, namely, we can consider skew fields having a valuation with noninteger values. This remark will be used in the following section in order to produce embeddings of free fields in Malcev-Neumann skew fields of arbitrary ordered groups.

The proof of the following result can be extracted from the proof of 3 , Theorem 1].

Theorem 2.1. Let $D$ be a skew field with infinite centre and let $K$ be a subfield of $D$ which is its own bicentralizer and whose centralizer $K^{\prime}$ in $D$ is such that the left $K$-space $K c K^{\prime}$ is infinite-dimensional, for all $c \in D^{\times}$. Suppose that $\nu: D \rightarrow \mathbb{R}_{\infty}$ is a valuation satisfying the condition that there exists a nonzero element $t$ of $K^{\prime}$ with $\nu(t)>0$. Then for every infinite countable set $\Sigma$ of full matrices over the free $D_{K}$-ring $D_{K}\langle X\rangle$ there exists a $\Sigma$-inverting homomorphism from $D_{K}\langle X\rangle$ into the completion $\widehat{D}$ of $D$ with respect to the valuation $\nu$.

(In particular, it follows that when $D$ and $X$ are infinite countable, $\nu$ is a discrete valuation and $\Sigma$ is the set of all full matrices over $D_{K}\langle X\rangle$ the completion $\widehat{D}$ contains the free field $D_{K}(\langle X\rangle)$. That is the statement of $[3$, Theorem 1(1)].)

The following consequence of Theorem 2.1 should be compared with [3, Corollary 1(1)].

Theorem 2.2. Let $D$ be a skew field with infinite centre $C$ such that the dimension of $D$ over $C$ is infinite. If there exists a nontrivial valuation $\nu: D \rightarrow \mathbb{R}_{\infty}$, then $\widehat{D}$ contains a free field $C(\langle X\rangle)$ on an infinite countable set $X$.

Proof. Let $X$ be an infinite countable set and let $F$ be an infinite countable subfield of $C$. Let $\Sigma$ denote the set of all full matrices over $F\langle X\rangle$. Since both $F$ and $X$ 
are infinite countable, $\Sigma$ is infinite countable. Moreover, $\Sigma$ is a set of full matrices over $D_{C}\langle X\rangle$ because the natural inclusion $F\langle X\rangle \hookrightarrow D_{C}\langle X\rangle$ is an honest map, by 6. Theorem 6.4.6]. It follows from Theorem 2.1 that there exists a $\Sigma$-inverting homomorphism $D_{C}\langle X\rangle \rightarrow \widehat{D}$ and, therefore, the composed map

$$
F\langle X\rangle \hookrightarrow D_{C}\langle X\rangle \longrightarrow \widehat{D}
$$

is a $\Sigma$-inverting $F$-ring homomorphism which extends to an $F$-ring homomorphism $F(\langle X\rangle) \rightarrow \widehat{D}$. Thus $X$ freely generates a free subfield of $\widehat{D}$ over $F$. Since $F$ is a central subfield of $D, X$ freely generates a free subfield of $\widehat{D}$ over the prime field of $D$ and, a fortiori, over $C$, by [3, Lemma 9].

This provides a more direct proof of the following version of [3, Corollary 1(2)].

Corollary 2.3. Let $K$ be a skew field with centre $F$ such that the dimension of $K$ over $F$ is infinite. Then the skew field of Laurent series $K((z))$ in $z$ over $K$ contains a free field $F(\langle X\rangle)$ on an infinite countable set $X$.

Proof. Let $\omega$ denote the valuation on $K(z)$ which extends the $z$-adic valuation on $K[z]$. Since there is a natural embedding $K \otimes_{F} F(z) \hookrightarrow K(z)$, it follows that $[K(z)$ : $F(z)] \geq\left[K \otimes_{F} F(z): F(z)\right]=[K: F]$ and, hence, $K(z)$ is infinite dimensional over $F(z)$, which is its (infinite) centre, by [6. Proposition 2.1.5]. So Theorem 2.2 applies and, therefore, $K((z))$ contains a free field $F(z)(\langle X\rangle)$ on an infinite countable set $X$. Finally, by [3, Lemma 9], $K((z))$ contains a free field $F(\langle X\rangle)$.

Now let $K$ be a skew field and let $(G,<)$ be a nontrivial ordered group with operation denoted multiplicatively. Given an element $z \in G$ with $z>1$, let $H$ denote the subgroup of $G$ generated by $z$. The subfield $K((H,<))$ of $K((G,<))$ is isomorphic to the Laurent series skew field $K((z))$. So, if the dimension of $K$ over its centre $F$ is infinite, Corollary 2.3 provides an embedding of the free field $F(\langle X\rangle)$ into $K((H,<))$ and, therefore, into $K((G,<))$. This proves the next result.

Corollary 2.4. Let $K$ be a skew field with centre $F$ such that the dimension of $K$ over $F$ is infinite and let $(G,<)$ be a nontrivial ordered group. Then the MalcevNeumann skew field $K((G,<))$ contains a free field $F(\langle X\rangle)$ on an infinite countable set $X$.

In the next section we shall see that most Malcev-Neumann skew fields contain free fields, even when the field of coefficients is not large compared with its centre.

\section{Free fields in MALCEV-Neumann SERIES RINGS}

In this section we consider arbitrary Malcev-Neumann skew fields containing crossed product rings. These fields will be shown to contain free fields very frequently. In particular, we shall see that Malcev-Neumann series over torsion-free nilpotent groups will have this property. Finally, some of the Malcev-Neumann skew fields considered will also be shown to be nonamenable in the sense of [8].

First we recall the construction of rings of Malcev-Neumann series containing crossed products. We follow [25, Chapter 1].

Given a skew field $K$ and a group $G$, a crossed product of $G$ over $K$ is an associative ring $K[G ; \sigma, \tau]$ which contains $K$ and which is a free right $K$-module with basis $\bar{G}=\{\bar{x}: x \in G\}$, a copy of $G$. Thus, each element $f \in K[G ; \sigma, \tau]$ is 
uniquely expressed as $f=\sum_{x \in G} \bar{x} a_{x}$, where $a_{x} \in K$, for all $x \in G$, and the support $\operatorname{supp} f=\left\{x \in G: a_{x} \neq 0\right\}$ of $f$ is finite. Here, multiplication satisfies

$$
\bar{x} \bar{y}=\overline{x y} \tau(x, y) \text { and } a \bar{x}=\bar{x} a^{\sigma(x)},
$$

for all $x, y \in G$ and $a \in K$, where $\tau: G \times G \rightarrow K^{\times}$and $\sigma: G \rightarrow \operatorname{Aut}(K)$ are maps. We can always assume that the identity element of $K[G ; \sigma, \tau]$ is $\overline{1}$ and that $K$ is a subfield of $K[G ; \sigma, \tau]$. If $H$ is a subgroup of $G$, then the crossed product $K\left[H ; \sigma_{\mid H}, \tau_{\mid H \times H}\right]$ embeds into $K[G ; \sigma, \tau]$. This subring will be denoted simply by $K[H ; \sigma, \tau]$.

Suppose that $(G,<)$ is an ordered group and that $K[G ; \sigma, \tau]$ is a crossed product of $G$ over a skew field $K$. Then we can define a new ring, denoted $K((G ; \sigma, \tau,<))$ and called Malcev-Neumann series ring, into which $K[G ; \sigma, \tau]$ embeds. The elements of $K((G ; \sigma, \tau,<))$ are uniquely expressed as $f=\sum_{x \in G} \bar{x} a_{x}$, with $a_{x} \in K$, for all $x \in G$, and $\operatorname{supp} f$ a well-ordered subset of $G$. Addition and multiplication are defined extending the ones in $K[G ; \sigma, \tau]$. Thus, given $f=\sum_{x \in G} \bar{x} a_{x}$ and $g=\sum_{x \in G} \bar{x} b_{x}$ in $K((G ; \sigma, \tau,<))$, addition and multiplication are given by

$$
f+g=\sum_{x \in G} \bar{x}\left(a_{x}+b_{x}\right), \quad f \cdot g=\sum_{x \in G} \bar{x}\left(\sum_{y z=x} \tau(y, z) a_{y}^{\sigma(z)} b_{z}\right) .
$$

It is a known fact that $K((G ; \sigma, \tau,<))$ is a skew field provided $K$ is $(22$, 24] $)$. If $H$ is a subgroup of $G$, the field $K\left(\left(H ; \sigma_{\mid H}, \tau_{\mid H \times H},<\right)\right)$ embeds into $K((G ; \sigma, \tau,<))$ and will be denoted by $K((H ; \sigma, \tau,<))$.

A few words are due on Malcev-Neumann series rings with regards to the invariance of their construction. First, we remark that a diagonal change of basis in $K[G ; \sigma, \tau]$ gives rise to the same series ring. Moreover, an order defined via an automorphism of $G$ which is induced by an automorphism of $K[G ; \sigma, \tau]$ will produce a Malcev-Neumann series ring which is isomorphic to $K((G ; \sigma, \tau,<))$.

The field of fractions of $K[G ; \sigma, \tau]$ inside $K((G ; \sigma, \tau,<))$, i.e. the subfield of $K((G ; \sigma, \tau,<))$ generated as a skew field by $K[G ; \sigma, \tau]$, will be denoted by $K(G ; \sigma, \tau)$. We remark that $K(G ; \sigma, \tau)$ does not depend on the order $<$ by [11, p. 183]. When we have a group ring $K[G]$, we will denote the Malcev-Neumann series ring by $K((G ;<))$ and the corresponding field of fractions of $K[G]$ by $K(G)$.

Now, let $K$ be a skew field and let $(G,<)$ be an ordered group which contains a noncyclic free subgroup $H$ on a set $X$. Let $K[G ; \sigma, \tau]$ be a crossed product and let $P$ denote the prime subfield of $K$. Because $H$ is a free group, after a diagonal change of basis, if necessary, it can be seen that the group algebra $P[H]$ is contained in $K[G ; \sigma, \tau]$ and, correspondingly, that $P((H,<))$ is contained in $K((G ; \sigma, \tau,<))$. By a result of Lewin [15], the field of fractions $P(H)$ of $P[H]$ inside $P((H,<))$ is isomorphic to the free field $P(\langle X\rangle)$ - so, in this case, we have an embedding of a free field into $K(G, \sigma, \tau) \subseteq K((G ; \sigma, \tau,<))$. We shall see below that MalcevNeumann series rings of a much larger class of ordered groups will have the property of containing free fields.

It is well known that the map $\omega: K((G ; \sigma, \tau,<)) \rightarrow G_{\infty}$ given by $\omega(0)=\infty$ and $\omega(f)=\min \operatorname{supp} f$, for all nonzero $f \in K((G ; \sigma, \tau,<))$, is a valuation of $K((G ; \sigma, \tau,<))$ with value group $G$. (Note that here we use multiplicative notation for the operation on $G$ and we shall do so whenever considering this natural valuation function.) If $\left(A,<^{\prime}\right)$ is another ordered group and $\pi:(G,<) \rightarrow\left(A,<^{\prime}\right)$ is a surjective order homomorphism, then $\nu: K((G ; \sigma, \tau,<)) \rightarrow A_{\infty}$, defined by 
$\nu(0)=\infty$ and $\nu(f)=\pi \omega(f)$, for all nonzero $f \in K((G ; \sigma, \tau,<))$, is a valuation on $K((G ; \sigma, \tau,<))$ with value group $A$.

In order to prove the existence of a free field inside $K((G ; \sigma, \tau,<))$ using Theorem 2.2. it is enough to establish for some subgroup $H$ of $G$ the infinite dimensionality of $K(H ; \sigma, \tau)$ over its centre and that $K((H ; \sigma, \tau,<))$ contains the completion $K \widehat{(H ; \sigma, \tau)}$ of $K(H ; \sigma, \tau)$ with respect to some valuation function on $K(H ; \sigma, \tau)$ with real values. The former is established in the next lemma which is proved in the same way as [3, Lemma 10], the latter is done in Lemma 3.2 .

Lemma 3.1. Let $K$ be a skew field and let $(G,<)$ be a nonabelian ordered group. Then any field of fractions of a crossed product of $G$ over $K$ is infinite dimensional over its centre.

Proof. Let $R=K[G ; \sigma, \tau]$ be a crossed product of $G$ over $K$, let $D$ be a field of fractions of $R$ and let $C$ be the centre of $D$.

We consider two cases. If $R$ is not an Ore domain, then, by 14, Proposition 10.25], it contains a noncommutative free algebra over its centre. This implies that $D$ contains a free subalgebra over $C$, by [21, Lemma 1], and, so, $[D: C]$ must be infinite.

If $R$ is an Ore domain, then $D$ is the Ore field of fractions of $R$, and, hence $D=K(G ; \sigma, \tau) \subseteq K((G ; \sigma, \tau,<))$.

Denote the centre of $G$ by $Z$. Now, since $R$ is an Ore domain, it is well-known that $K[Z ; \sigma, \tau]$ must also be an Ore domain and, so, the Ore field of fractions $K(Z ; \sigma, \tau)$ of $K[Z ; \sigma, \tau]$ is contained in $D$. We shall see, first, that $C \subseteq K((Z ; \sigma, \tau,<))$.

Indeed, let $f \in C$ and express $f$ as a series in $K((G ; \sigma, \tau,<))$, say $f=\sum_{x \in G} \bar{x} a_{x}$. For each $y \in G$, we have $\bar{y} f=f \bar{y}$. Thus,

$$
\sum_{x \in G} \overline{y x} \tau(y, x) a_{x}=\bar{y} f=f \bar{y}=\sum_{x \in G} \overline{x y} \tau(x, y) a_{x}^{\sigma(y)} .
$$

Since $y(\operatorname{supp} f)=\operatorname{supp}(\bar{y} f)=\operatorname{supp}(f \bar{y})=(\operatorname{supp} f) y$, we get that $\operatorname{supp} f \subseteq Z$. Hence $C \subseteq K((Z ; \sigma, \tau,<))$.

Now, it is known that $G / Z$ is an orderable group (see, e.g. [2, Theorem 2.24]). Since $G$ is not abelian, $G / Z$ is torsion free. If $x \in G \backslash Z$, then $\left\{\bar{x}^{n}: n \in \mathbb{Z}, n \geq 0\right\}$ is linearly independent over $K((Z ; \sigma, \tau,<))$ inside $K((G ; \sigma, \tau,<))$, and, therefore, over $C$. Since $\bar{x} \in D$, we get that $[D: C]=\infty$, as desired.

Lemma 3.2. Let $(G,<)$ be an ordered group, let $K$ be a skew field and let $K[G ; \sigma, \tau]$ be a crossed product of $G$ over $K$. Suppose that $\pi: G \rightarrow A$ is a surjective homomorphism of ordered groups, where $A$ is a nontrivial subgroup of the additive group $\mathbb{R}$ of the real numbers. Then $K((G ; \sigma, \tau,<))$ is complete with respect to the valuation $\nu=\pi \omega: K((G ; \sigma, \tau,<)) \rightarrow A_{\infty}$.

Proof. Fix $c \in(0,1)$ and consider the metric $d$ in $K((G ; \sigma, \tau,<))$ induced by $\nu$, i.e. $d\left(f_{1}, f_{2}\right)=c^{\nu\left(f_{1}-f_{2}\right)}$ for $f_{1}, f_{2} \in K((G ; \sigma, \tau,<))$.

Let $\left(u_{n}\right)_{n \geq 1}$ be a Cauchy sequence in $K((G ; \sigma, \tau,<))$, say $u_{n}=\sum_{x \in G} \bar{x} a_{x}^{(n)}$ where $a_{x}^{(n)} \in K$. For each $k \in \mathbb{N}$ choose $n_{k} \in \mathbb{N}$ such that $d\left(u_{p}, u_{q}\right)<c^{k}$ whenever $p, q \geq n_{k}$, satisfying $n_{0}<n_{1}<n_{2}<\cdots<n_{k}<\cdots$. Observe that for $k, p, q \in \mathbb{N}$,

$$
d\left(u_{p}, u_{q}\right)<c^{k} \text { if and only if } a_{x}^{(p)}=a_{x}^{(q)} \text { for all } x \text { with } \pi(x) \leq k .
$$

For each $x \in G$, let $k=k(x)$ be the smallest $k \in \mathbb{N}$ such that $\pi(x) \leq k$. Define $a_{x}=a_{x}^{\left(n_{k}\right)}$ and let $u=\sum_{x \in G} \bar{x} a_{x}$. 
First we show that $u \in K((G ; \sigma, \tau,<))$, that is, we show that $\operatorname{supp} u$ is wellordered. Indeed, fix $y \in G$. Note that if $x \leq y$, then $\pi(x) \leq \pi(y)$ because $\pi$ is a homomorphism of ordered groups. Let $k$ and $l$ be the smallest natural numbers such that $\pi(x) \leq k$ and $\pi(y) \leq l$, respectively. Then $n_{k} \leq n_{l}$, and therefore $a_{x}=a_{x}^{\left(n_{k}\right)}=a_{x}^{\left(n_{l}\right)}$ because $d\left(u_{n_{k}}, u_{n_{l}}\right)<c^{k}$. Hence $\left\{x: x \leq y, a_{x} \neq 0\right\} \subseteq \operatorname{supp} u_{n_{l}}$. Let $S$ be a nonempty subset of $\operatorname{supp} u$ and let $y \in S$. Let $l$ be the smallest natural such that $\pi(y) \leq l$. We have that $\{x \in S: x \leq y\} \neq \emptyset$ and, by the foregoing argument, it is contained in $\operatorname{supp} u_{n_{l}}$. Thus $S$ has a least element. Therefore supp $u$ is well-ordered.

Finally, we show that $\lim u_{n}=u$. Given $\varepsilon>0$, there exists $k \in \mathbb{N}$ such that $c^{k}<\varepsilon$. Choose $n>n_{k}$. Then,

$$
\begin{aligned}
u-u_{n} & =\sum_{x \in G} \bar{x} a_{x}-\sum_{x \in G} \bar{x} a_{x}^{(n)} \\
& =\sum_{\{x: \pi(x) \leq k\}} \bar{x} a_{x}-\sum_{\{x: \pi(x) \leq k\}} \bar{x} a_{x}^{(n)}+\sum_{\{x: \pi(x)>k\}} \bar{x} a_{x}-\sum_{\{x: \pi(x)>k\}} \bar{x} a_{x}^{(n)} \\
& =\sum_{\{x: \pi(x)>k\}} \bar{x} a_{x}-\sum_{\{x: \pi(x)>k\}} \bar{x} a_{x}^{(n)} .
\end{aligned}
$$

Hence $d\left(u, u_{n}\right)=c^{\nu\left(u-u_{n}\right)}<c^{k}<\varepsilon$, as desired.

Now we can state and prove the main result in this section.

Theorem 3.3. Let $(G,<)$ be an ordered group and let $K$ be a skew field. Consider a crossed product $K[G ; \sigma, \tau]$ and its Malcev-Neumann series ring $D=K((G ; \sigma, \tau,<))$. If there exists a convex jump $(N, H)$ of $G$ such that the centre $F$ of $K(H ; \sigma, \tau)$ is infinite and $K(H ; \sigma, \tau)$ is infinite-dimensional over $F$, then $D$ contains a free field $C(\langle X\rangle)$, where $X$ is an infinite countable set and $C$ is the centre of $K(G ; \sigma, \tau)$.

Proof. The canonical projection $\pi: H \rightarrow H / N$ is a surjective homomorphism of ordered groups and $H / N$ is a nontrivial subgroup of $\mathbb{R}$. By Lemma $3.2, K((H ; \sigma, \tau,<))$ is complete with respect to the valuation $\nu=\pi \omega$. Thus, the completion $K \widehat{(H ; \sigma, \tau)}$ of $K(H ; \sigma, \tau)$ with respect to $\nu$ is contained in $K((H ; \sigma, \tau,<))$. Hence Theorem 2.2 implies that the free field $F(\langle X\rangle)$ is contained in $K((H ; \sigma, \tau,<)) \subseteq D$.

Let $P$ denote the prime subfield of $K(H ; \sigma, \tau)$. Then the free field $P(\langle X\rangle)$ is contained in $F(\langle X\rangle) \subseteq D$ by [6, Theorem 6.4.6]. Now, by [3, Lemma 9], we get that $C(\langle X\rangle)$ is contained in $D$.

When the crossed product is a group ring, we can find some explicit conditions which imply the existence of a convex jump satisfying the hypothesis of the above theorem, as the next corollary shows.

Corollary 3.4. Let $(G,<)$ be a nonabelian ordered group and let $K$ be a skew field with centre $F$. Consider the group ring $K[G]$ and its Malcev-Neumann series ring $K((G ;<))$. If

(a) $F$ is infinite, or

(b) the centre of $G$ is nontrivial, then $K((G ;<))$ contains a free field $C(\langle X\rangle)$, where $X$ is an infinite countable set and $C$ is the centre of $K(G)$. 
Proof. Let $x, y \in G$ be such that $x>1, y>1$ and $x y \neq y x$. In case (国), set $z=1$; in case (b), let $z$ be an element of the centre of $G$ satisfying $z>1$. Let $w=\max \{x, y, z\}$ and let $(N, H)$ be the convex jump associated to $w$, that is, $N$ is the union of all convex subgroups which do not contain $w$ and $H$ the intersection of all convex subgroups which contain $w$. In particular, $H$ is a subgroup of $G$ containing $x, y, z$. Let $E$ denote the centre of $K(H)$. Since $H$ is not abelian, Lemma 3.1 implies that $[K(H): E]$ is infinite. Since $F[z] \subseteq E$, we get that $E$ is

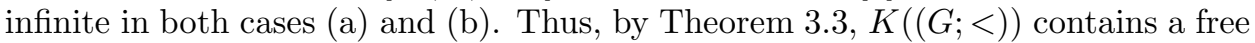
field $C(\langle X\rangle)$ on an infinite countable set $X$.

The following is an example in which the conditions of the above corollary are satisfied.

Example 3.5. Torsion-free nilpotent groups are known to be orderable. Moreover, a nonabelian torsion-free nilpotent group has a nontrivial centre. It follows from Corollary 3.4 that the Malcev-Neumann series ring of such a group over a skew field contains a free subfield. For instance, if $G$ is a finitely generated torsion-free nilpotent group, then $G$ has a central series

$$
G=F_{1} \supseteq F_{2} \supseteq F_{3} \supseteq \cdots \supseteq F_{r} \supseteq F_{r+1}=\{1\},
$$

in which $F_{i} / F_{i+1}$ is an infinite cyclic group for all $i=1, \ldots, r$ (see e.g. [12]). Letting $f_{i}$ be a representative in $G$ of a generating element of $F_{i} / F_{i+1}$, it follows that the elements of $G$ can be written in a unique way in the form $f_{1}^{\alpha_{1}} f_{2}^{\alpha_{2}} \ldots f_{r}^{\alpha_{r}}$, with $\alpha_{i} \in \mathbb{Z}$. Since $\left[F_{j}, F_{i}\right] \subseteq F_{j+1}$, we have $f_{j}^{\alpha_{j}} f_{i}^{\alpha_{i}}=f_{i}^{\alpha_{i}} f_{j}^{\alpha_{j}} f_{j+1}^{\gamma_{j+1}} \ldots f_{r}^{\gamma_{r}}$, for some $\gamma_{j+1}, \ldots, \gamma_{r} \in \mathbb{Z}$, whenever $j>i$. Therefore, $G$ can be lexicographically ordered, that is, $G$ is an ordered group with order < defined in the following way: $f_{1}^{\alpha_{1}} f_{2}^{\alpha_{2}} \ldots f_{r}^{\alpha_{r}}<f_{1}^{\beta_{1}} f_{2}^{\beta_{2}} \ldots f_{r}^{\beta_{r}}$ if and only if there exists some $s$ for which $\alpha_{s}<\beta_{s}$, while $\alpha_{i}=\beta_{i}$ for all $i=1, \ldots, s-1$. Now, given a skew field $K$, it is known that the group ring $K[G]$ is an Ore domain with field of fractions $D$, say, which must therefore be a subfield of $K((G ;<))$. When $G$ is nonabelian, $K((G ;<))$ contains a free field $C(\langle X\rangle)$ on an infinite countable set $X$ over the centre $C$ of $D$.

Finally, it should be remarked that $G$ can be ordered in a number of different ways. For example, if $G$ is the free nilpotent group of class 2 generated by two elements,

$$
G=\langle x, y:[[x, y], x]=[[x, y], y]=1\rangle,
$$

then there is an infinite number of ways of ordering $G /\langle[x, y]\rangle \cong \mathbb{Z} \times \mathbb{Z}$ (see $[23$, Example 1.1]). Since $[x, y]$ is central, each one of these orderings can be lifted to an order on $G$, and some of them do not come from a central series with infinite cyclic quotients. Corollary 3.4 though, guarantees the presence of free fields in $K((G ;<))$ whatever order $<$ is taken.

We shall look at this example again in Section 5

In 8, the concept of amenable skew fields was introduced. We now show that Malcev-Neumann series rings provide examples of nonamenable skew fields.

Following [8, let $F$ be a commutative field and let $A$ be an $F$-algebra. We say that $A$ is amenable over $F$ if for any finite subset $\left\{r_{1}, \ldots, r_{n}\right\} \subseteq A$ and real number $\epsilon>0$ there exists a finite-dimensional $F$-subspace $V$ of $A$ such that

$$
\frac{\operatorname{dim}_{F}\left(\sum_{i=1}^{n} r_{i} V\right)}{\operatorname{dim}_{F} V}<1+\epsilon
$$


We begin by remarking that amenability is preserved under ground field extensions.

Lemma 3.6. Let $F$ be a commutative field and let $K$ be a commutative field extension of $F$. If $A$ is an amenable $F$-algebra, then $K \otimes_{F} A$ is an amenable $K$-algebra.

Proof. Let $\left\{r_{1}, \ldots, r_{n}\right\} \subseteq K \otimes_{F} A$ and $\epsilon>0$. Suppose that $r_{i}=\sum_{l=1}^{n_{i}} e_{i l} \otimes a_{i l}$. Since $A$ is amenable over $F$, there exists a finite dimensional $F$-subspace $V$ of $A$ such that

Let $W=K \otimes_{F} V$. Then,

$$
\frac{\operatorname{dim}_{F}\left(\sum_{i=1}^{n} \sum_{l=1}^{n_{i}} a_{i l} V\right)}{\operatorname{dim}_{F} V}<1+\epsilon .
$$

$$
\begin{aligned}
\frac{\operatorname{dim}_{K}\left(\sum_{i=1}^{n} r_{i} W\right)}{\operatorname{dim}_{K} W} & \leq \frac{\operatorname{dim}_{K}\left(\sum_{i=1}^{n} \sum_{l=1}^{n_{i}}\left(1 \otimes a_{i l}\right) W\right)}{\operatorname{dim}_{K} W} \\
& =\frac{\operatorname{dim}_{F}\left(\sum_{i=1}^{n} \sum_{l=1}^{n_{i}} a_{i l} V\right)}{\operatorname{dim}_{F} V} \\
& <1+\epsilon,
\end{aligned}
$$

as desired.

Proposition 3.7. Let $F$ be a commutative field, let $K$ be a commutative field extension of $F$ and let $X$ be a set with $|X|>1$. If $F(\langle X\rangle)$ is amenable over $F$, then $K(\langle X\rangle)$ is amenable over $K$.

Proof. Let $\mu: K \otimes_{F} F(\langle X\rangle) \rightarrow K(\langle X\rangle)$ denote the multiplication map. Since $F(\langle X\rangle)$ is a simple algebra with centre $F$ (by [6, Theorem 5.5.11]), $K \otimes_{F} F(\langle X\rangle)$ is simple. Hence, $\mu$ is an injective homomorphism of $K$-algebras. By Lemma 3.6 . $K \otimes_{F} F(\langle X\rangle)$ is amenable over $K$. By [8, Lemma 2.1], $K \otimes_{F} F(\langle X\rangle)$ is a right Ore domain and its Ore field of fractions $K \widehat{\otimes_{F} F(\langle X\rangle)}$ is amenable over $K$. But $\left.K \widehat{\otimes_{F} F(}\langle X\rangle\right)$ is a subfield of $K(\langle X\rangle)$ that contains both $K$ and $X$. Thus $K \widehat{\otimes_{F} F(\langle X\rangle)}=$ $K(\langle X\rangle)$. Therefore $K(\langle X\rangle)$ is amenable over $K$.

Corollary 3.8. Let $F$ be a subfield of the field of complex numbers $\mathbb{C}$ and let $X$ be a set with $|X|>1$. Then $F(\langle X\rangle)$ is not amenable over any subfield $L$ of $F$.

Proof. By $[8$, Theorem $1(\mathrm{e})], \mathbb{C}(\langle X\rangle)$ is not amenable over $\mathbb{C}$. It follows from Proposition 3.7 that $L(\langle X\rangle)$ is not amenable over $L$ for any subfield $L$ of $\mathbb{C}$. If $L \subseteq F$, then $L(\langle X\rangle) \subseteq F(\langle X\rangle)$, by [6. Theorem 6.4.6]. It follows from [8, Proposition 3.1], that $F(\langle X\rangle)$ is not amenable over $L$.

We are now ready to show that, under mild conditions, Malcev-Neumann series rings are nonamenable skew fields.

Corollary 3.9. Let $F$ be a subfield of the field of complex numbers $\mathbb{C}$. Let $K$ be a skew field with centre $Z$, let $(G,<)$ be an ordered group and let $K[G ; \sigma, \tau]$ be a crossed product which is an $F$-algebra. If either

(a) $G$ is nonabelian, or

(b) $G$ is abelian, $[K: Z]=\infty$ and $K[G ; \sigma, \tau]=K[G]$,

then the Malcev-Neumann series ring $K((G ; \sigma, \tau,<))$ is not an amenable skew field over any subfield $L$ of $F$. 
Proof. Because $G$ is ordered, the only units in $K[G ; \sigma, \tau]$ are the trivial ones. Hence, the fact that $K[G ; \sigma, \tau]$ is an $F$-algebra implies that $F \subseteq Z$. In case (国), let $(N, H)$ be a convex jump of $(G,<)$ with $H$ nonabelian. Let $E$ denote the centre of $K(H ; \sigma, \tau)$. Then $F \subseteq E$ and, thus, $E$ is infinite. By Lemma $3.1[K(H ; \sigma, \tau): E]$ is infinite. Hence, Theorem 3.3 yields that $K((G ; \sigma, \tau,<))$ contains a free field $C(\langle X\rangle)$, where $X$ is an infinite countable set and $C$ is the centre of $K(G ; \sigma, \tau)$. Since $F \subseteq C$, [6. Theorem 6.4.6] implies that $F(\langle X\rangle) \subseteq C(\langle X\rangle)$. In case (b), by Corollary 2.4 $K((G,<))$ contains a free field $Z(\langle X\rangle)$, where $X$ is an infinite countable set. Again, by [6. Theorem 6.4.6], $Z(\langle X\rangle)$ contains $F(\langle X\rangle)$.

In both cases, $K((G ; \sigma, \tau,<))$ contains a free field $F(\langle X\rangle)$, for infinite countable set $X$. By Corollary [3.8, $F(\langle X\rangle)$ is not amenable over any subfield $L$ of $F$. It follows from [8, Proposition 3.1] that $K((G ; \sigma, \tau,<))$ is not an amenable skew field over $L$.

As a consequence, we note that if $K$ is a skew field of zero characteristic and $(G,<)$ is a nonabelian ordered group, then $K((G ; \sigma, \tau,<))$ is a nonamenable skew field over $\mathbb{Q}$.

\section{Skew Laurent series}

In this section we show that fields of fractions of skew polynomial rings over skew fields are endowed with natural valuations whose completions, skew Laurent series rings, contain free fields. In particular, it will be shown that the Weyl field can be embedded in a complete skew field containing a free field.

In the absence of derivations, the skew fields considered in this section can be treated by the methods of the previous section. We, therefore, concentrate in the case where a derivation is present.

Let $A$ be a ring, let $\sigma$ be an endomorphism of $A$ and let $\delta$ be a (right) $\sigma$-derivation of $A$, that is, $\delta: A \rightarrow A$ is an additive map satisfying

$$
\delta(a b)=\delta(a) \sigma(b)+a \delta(b),
$$

for all $a, b \in A$. The skew polynomial ring in $x$ over $A$, hereafter denoted by $A[x ; \sigma, \delta]$, is the free right $A$-module on the nonnegative powers of $x$ with multiplication induced by

$$
a x=x \sigma(a)+\delta(a)
$$

for all $a \in A$. If $A$ is a skew field then $A[x ; \sigma, \delta]$ is a principal right ideal domain and its field of fractions will be denoted by $A(x ; \sigma, \delta)$. When $A$ is a right Ore domain with field of fractions $Q(A)$ and $\sigma$ is injective, $\sigma$ and $\delta$ can be extended to $Q(A)$ and we can consider the skew polynomial ring $Q(A)[x ; \sigma, \delta]$. In this case we have

$$
A[x ; \sigma, \delta] \subseteq Q(A)[x ; \sigma, \delta] \subseteq Q(A)(x ; \sigma, \delta)
$$

and so $A[x ; \sigma, \delta]$ is a right Ore domain with field of fractions $Q(A)(x ; \sigma, \delta)$.

For an arbitrary endomorphism $\sigma$ of the skew field $K$ a further construction will be considered. The ring of skew power series $K[[x ; \sigma]]$ is defined to be the set of power series on $x$ of the form $\sum_{i=0}^{\infty} x^{i} a_{i}$, with $a_{i} \in K$, where addition is done coefficientwise and multiplication is given by the rule $a x=x \sigma(a)$, for $a \in K$. When $\sigma$ is an automorphism, we can embed $K[[x ; \sigma]]$ into the ring of skew Laurent series $K((x ; \sigma))$ whose elements are series $\sum_{-r}^{\infty} x^{i} a_{i}$, with $r$ a nonnegative integer. In $K((x ; \sigma))$, multiplication satisfies $a x^{n}=x^{n} \sigma^{n}(a)$, for every integer $n$ and $a \in K$. It is well known that $K((x ; \sigma))$ is a skew field containing $K(x ; \sigma)$. 
As mentioned in [6. Section 2.3], when $\delta \neq 0$ we must look at a more general construction. Given an automorphism $\sigma$ of a skew field $K$ and a $\sigma$-derivation $\delta$ on $K$, denote by $R$ the ring of all power series $\sum_{i=0}^{\infty} a_{i} y^{i}$ on $y$ with multiplication induced by $y a=\sum_{i=0}^{\infty} \sigma \delta^{i}(a) y^{i+1}$, for $a \in K$. In [6, Theorem 2.3.1] it is shown that $R$ is a domain and $S=\left\{1, y, y^{2}, \ldots\right\}$ is a left Ore set in $R$ whose localization $S^{-1} R$ is a skew field, consisting of all skew Laurent series $\sum_{i=r}^{\infty} a_{i} y^{i}$, with $r \in \mathbb{Z}$. Because in $S^{-1} R$ we have $y a=\sigma(a) y+y \delta(a) y$, for all $a \in K$, it follows that $a y^{-1}=y^{-1} \sigma(a)+$ $\delta(a)$. Hence, the subring generated by $y^{-1}$ in $S^{-1} R$ is an ordinary skew polynomial ring on $y^{-1}$. Because of that we shall adopt the notation $R=K\left[\left[x^{-1} ; \sigma, \delta\right]\right]$ and $S^{-1} R=K\left(\left(x^{-1} ; \sigma, \delta\right)\right)$. So we have an embedding $K[x ; \sigma, \delta] \subseteq K\left(\left(x^{-1} ; \sigma, \delta\right)\right)$, sending $x$ to $y^{-1}$ and, hence, $K(x ; \sigma, \delta) \subseteq K\left(\left(x^{-1} ; \sigma, \delta\right)\right)$. It is also clear that $K\left(\left(x^{-1} ; \sigma, \delta\right)\right)=K\left[\left[x^{-1} ; \sigma, \delta\right]\right]+K[x ; \sigma, \delta]$.

The field $K(x ; \sigma, \delta)$ has a discrete valuation "at infinity" $\nu$ which extends the valuation - deg on $K[x ; \sigma, \delta]$, where deg denotes the usual degree function on $K[x ; \sigma, \delta]$, that is, $\operatorname{deg}\left(\sum x^{i} a_{i}\right)=\max \left\{i: a_{i} \neq 0\right\}$.

Lemma 4.1. Let $K$ be a skew field with an automorphism $\sigma$ and a $\sigma$-derivation $\delta$. Then the map $\omega: K\left(\left(x^{-1} ; \sigma, \delta\right) \rightarrow \mathbb{Z}_{\infty}\right.$ defined by

$$
\omega(f)=\sup \left\{n: f \in K\left[\left[x^{-1} ; \sigma, \delta\right]\right] y^{n}\right\},
$$

is a valuation on $K\left(\left(x^{-1} ; \sigma, \delta\right)\right)$ which extends the valuation $\nu$ on $K(x ; \sigma, \delta)$. Moreover, $K\left(\left(x^{-1} ; \sigma, \delta\right)\right)$ is the completion of $K(x ; \sigma, \delta)$.

Proof. To show that $\omega$ is a valuation, the only nonobvious fact to be proved is that if $f$ and $g$ are nonzero elements of $K\left(\left(x^{-1} ; \sigma, \delta\right)\right)$, then $\omega(f g)=\omega(f)+\omega(g)$. This follows from the fact that, since $\sigma$ is invertible, it is possible to write, for every integer $n$ and $a \in K, y^{n} a=\sigma^{n}(a) y^{n}+h y^{n+1}$, for some $h \in K\left[\left[x^{-1} ; \sigma, \delta\right]\right]$.

Clearly, $\omega_{\mid K(x ; \sigma, \delta)}=\nu$. That $K(x ; \sigma, \delta)$ is dense in $K\left(\left(x^{-1} ; \sigma, \delta\right)\right)$ follows from the fact that every Laurent series $\sum_{i=n}^{\infty} a_{i} y^{i}$ in $K\left(\left(x^{-1} ; \sigma, \delta\right)\right)$ is the limit of its partial sums, which are elements in $K(x ; \sigma, \delta)$. It remains to prove that $K\left(\left(x^{-1} ; \sigma, \delta\right)\right)$ is complete. Let $\left(u_{n}\right)$ be a Cauchy sequence in $K\left(\left(x^{-1} ; \sigma, \delta\right)\right)$, say

$$
u_{n}=\sum_{i \geq m_{n}} a_{i}^{(n)} y^{i},
$$

with $a_{i}^{(n)} \in K$ and $m_{n} \in \mathbb{Z}$. We proceed as in the proof of Lemma 3.2. For each $k \in \mathbb{Z}$ choose nonnegative integers $n_{k}$ satisfying $n_{0}<n_{1}<n_{2}<\ldots$ such that $\omega\left(u_{p}-u_{q}\right)>k$, for all $p, q \geq n_{k}$. Consider the following element of $K\left(\left(x^{-1} ; \sigma, \delta\right)\right)$,

$$
u=\sum_{i<0} a_{i}^{\left(n_{0}\right)} y^{i}+\sum_{i \geq 0} a_{i}^{\left(n_{i}\right)} y^{i} .
$$

For all $k, \omega\left(u_{n_{k}}-u\right) \geq k+1$ and, hence, the subsequence $\left(u_{n_{k}}\right)$ of $\left(u_{n}\right)$ converges to $u$. Since $\left(u_{n}\right)$ is a Cauchy sequence, $\left(u_{n}\right)$ converges to $u$. It follows that $K\left(\left(x^{-1} ; \sigma, \delta\right)\right)$ is complete.

In the case that $\delta=0, K(x ; \sigma)$ has an $x$-adic discrete valuation $\eta$, defined by $\eta\left(f g^{-1}\right)=o(f)-o(g)$, for all $f, g \in K[x ; \sigma], g \neq 0$, where $o\left(\sum x^{i} a_{i}\right)=\min \left\{i: a_{i} \neq\right.$ $0\}$ is the order function on $K[x ; \sigma]$, which is a valuation. Denote by $\zeta$ the integer valued function on $K((x ; \sigma))$ defined by $\zeta(h)=\sup \left\{n: h \in x^{n} K[[x ; \sigma]]\right\}$, for all $h \in K((x ; \sigma)), h \neq 0$. It is easy to see that $\zeta$ is a discrete valuation on $K((x ; \sigma))$ 
which coincides with $\eta$ on $K(x ; \sigma)$. With a proof similar to the one of Lemma 4.1 it can be shown that $K((x ; \sigma))$ is the completion of $K(x ; \sigma)$ with respect to $\eta$.

Our next aim is to guarantee the existence of free fields in completions of skew rational function fields. For that, we recall the following definition. An automorphism $\sigma$ in a ring is said to have inner order $r$ if $\sigma^{r}$ is the least positive power of $\sigma$ which is inner. If $\sigma^{r}$ is not inner for any $r>0$, then $\sigma$ is said to have infinite inner order.

Theorem 4.2. Let $K$ be a skew field with centre $F$, let $\sigma$ be an automorphism of $K$ and let $\delta$ be a $\sigma$-derivation of $K$. Suppose that $K[x ; \sigma, \delta]$ is simple or that $\sigma$ has infinite inner order. If $\delta \neq 0$, let $D=K\left(\left(x^{-1} ; \sigma, \delta\right)\right)$, otherwise, let $D=K((x ; \sigma))$. If the field $F_{0}=\{c \in F: \sigma(c)=c$ and $\delta(c)=0\}$ is infinite, then $D$ contains a free field $F_{0}(\langle X\rangle)$ on an infinite countable set $X$.

Proof. Since $K[x ; \sigma, \delta]$ is simple or $\sigma$ has infinite inner order, it follows from [6, Theorem 2.2.10] that the centre of $K(x ; \sigma, \delta)$ coincides with $F_{0}$. The set $\left\{x^{i}: i \in \mathbb{Z}\right\}$ is linearly independent over $F_{0}$, because it is over $K$; hence $K(x ; \sigma, \delta)$ is infinite dimensional over its infinite centre $F_{0}$. By Theorem 2.2, $D$ contains a free field on an infinite countable set over $F_{0}$, for, as we have seen above, it is the completion of $K(x ; \sigma, \delta)$ with respect to appropriate discrete valuations.

We remark that the case $\delta=0$ in the above theorem had already been considered in [3, Theorem 4] for a commutative field $K$ of coefficients.

An example of a skew polynomial ring with a nonzero derivation is the first Weyl algebra over a skew field $K$,

$$
A_{1}(K)=K\left\langle x_{1}, x_{2}: x_{1} x_{2}-x_{2} x_{1}=1\right\rangle=K\left[x_{1}\right]\left[x_{2} ; I, \frac{d}{d x_{1}}\right],
$$

where $I$ above stands for the identity automorphism of $K\left[x_{1}\right]$. It is well known that $A_{1}(K)$ is an Ore domain with field of fractions $Q_{1}(A)=K\left(x_{1}\right)\left(x_{2} ; I, \frac{d}{d x_{1}}\right)$, called the Weyl field.

Corollary 4.3. Let $K$ be a skew field with centre $F$. If char $K=0$ then the field of skew Laurent series $K\left(x_{1}\right)\left(\left(x_{2}^{-1} ; I, \frac{d}{d x_{1}}\right)\right)$ contains a free field $F(\langle X\rangle)$, where $X$ is an infinite countable set.

Proof. Let $B=K\left(x_{1}\right)\left[x_{2} ; I, \frac{d}{d x}\right]$. Then $A_{1}(K) \subseteq B \subseteq K\left(x_{1}\right)\left(x_{2} ; I, \frac{d}{d x_{1}}\right)$ and $Q(B)=Q_{1}(A)=K\left(x_{1}\right)\left(x_{2} ; I, \frac{d}{d x_{1}}\right)$. Clearly, $\frac{d}{d x_{1}}$ is not an inner derivation. It follows from [13, Corollary 3.16] that $B$ is a simple ring. Since char $K=0$, we have $Z\left(K\left(x_{1}\right)\right)_{0}=\left\{f \in Z\left(K\left(x_{1}\right)\right): \frac{d}{d x_{1}}(f)=0\right\}=F\left(x_{1}\right)_{0}=F$, an infinite field. By Theorem 4.2 the field of skew Laurent series $K\left(x_{1}\right)\left(\left(x_{2}^{-1} ; I, \frac{d}{d x_{1}}\right)\right)$ contains a free field on an infinite countable set over $F$.

A final word will be given on a related problem, that of division rings generated by Lie algebras. Given a nonabelian Lie algebra $L$ over a commutative field $k$, its universal enveloping algebra $U(L)$ has a natural filtration given by the powers of $L$ inside $U(L)$, and this filtration defines a valuation $\nu$ on $U(L)$. (See [6, Section 2.5] or [17.) By [17, Theorem 2], $U(L)$ has a field of fractions $D(L)$ with a valuation extending $\nu$. Theorem 2.2, then, implies that if char $k=0$ the completion $\overline{D(L)}$ of $D(L)$ contains a free field $C(\langle X\rangle)$, for an infinite countable set $X$, where $C$ stands for the centre of $D(L)$. Note that Theorem 2.2 can be applied in this setting because, by [18, $D(L)$ contains a free subalgebra. 


\section{Some SKEW FIELDS THAT DO NOT CONTAIN FREE FIELDS}

In this section we shall present a theorem giving a necessary condition for a division ring to contain a free field. This will then be used in order to produce examples of division rings which, although containing free algebras, do not contain free fields.

The results in this section are due to A. Lichtman, but have not been published before. The authors are indebted to him for having kindly permitted this material to be included in the paper.

Throughout this section, $k$ will denote a commutative field.

Let $A$ be a $k$-algebra. Denote by $A^{\text {op }}$ the opposite algebra of $A$. Regard the $(A, A)$-bimodule $A$ as a left module over the enveloping algebra $A \otimes_{k} A^{\text {op }}$ in the usual way, that is, via $a \otimes b \cdot x=a x b$, for all $a, b, x \in A$. We shall make use of the following result of Sweedler [26].

Lemma 5.1. Let $D$ be a skew field which is a k-algebra. If $D \otimes_{k} D^{\text {op }}$ is a left noetherian algebra, then $D$ does not contain an infinite strictly ascending chain of subfields $D_{1} \varsubsetneqq D_{2} \varsubsetneqq D_{3} \varsubsetneqq \ldots$ which are $k$-subalgebras.

For a direct proof we refer to [1].

As a consequence we obtain the following necessary condition for a skew field to contain a free field.

Theorem 5.2. Let $D$ be a skew field and let $k$ be a central subfield of $D$. If $D$ contains a free field $k(\langle x, y\rangle)$ then $D \otimes_{k} D^{\text {op }}$ cannot be a left noetherian ring.

Proof. If $D$ contains the free field $k(\langle x, y\rangle)$ then, by [6, Corollary 5.5.9], $D$ contains a free field with an infinite countable number of generators, say, $k\left(\left\langle x_{1}, x_{2}, \ldots\right\rangle\right) \subseteq$ $D$. For every $n \geq 1$, letting $D_{n}=k\left(\left\langle x_{1}, \ldots, x_{n}\right\rangle\right)$, we obtain an infinite strictly ascending chain of subfields in $D, D_{1} \varsubsetneqq D_{2} \varsubsetneqq \ldots$ It follows from Lemma 5.1 that $D \otimes_{k} D^{\text {op }}$ is not a left noetherian ring.

Now consider a nonabelian torsion-free polycyclic-by-finite group $G$. We recall that the group ring $K[G]$ is a noetherian domain for every skew field $K$ (cf. [25, Proposition 1.6 and Corollary 37.11]). By Theorem 5.2 we have the following result.

Corollary 5.3. The field of fractions of the group algebra of a nonabelian torsionfree polycyclic-by-finite group over a commutative field does not contain a noncommutative free field (over any subfield).

Proof. Let $G$ be a nonabelian torsion-free polycyclic-by-finite group. Let $F$ denote the left classic field of fractions of $k[G]$ and consider the $k$-algebra $R=k[G] \otimes_{k} F^{\text {op }} \cong$ $F^{\mathrm{op}}[G]$. Since $k[G]$ is a left Ore domain, $T=(k[G] \backslash\{0\}) \otimes_{k} 1$ is a left denominator subset of $R$. Since $R$ is left noetherian, $T^{-1} R \cong F \otimes_{k} F^{\text {op }}$ is also left noetherian. By Theorem [5.2, $F$ does not contain a free field over $k$. Therefore, $F$ does not contain a free field over any central subfield, by [3, Lemma 9]. Consequently, $F$ does not contain a free field over any subfield, by [4, Corollary p. 114] and [6, Theorem 5.8.12].

Let $H$ be a nonabelian torsion-free finitely generated nilpotent group and consider the group algebra $k[H]$. Since $H$ is polycyclic-by-finite then the group algebra $k[H]$ is a noetherian domain. In [20, Makar-Limanov showed that the field of fractions of $k[H]$ contains noncommutative free algebras over $k$ and, consequently, it 
contains fields of fractions of free algebras. However, none of these fields of fractions is a free field, by Corollary 5.3 .

Proceeding in a similar fashion, it is possible to show that the field of fractions $Q$ of the first Weyl algebra over a commutative field $k$ does not contain a noncommutative free field (over any subfield). We remark that, again, Makar-Limanov proved in [19] that if $k$ has zero characteristic, $Q$ contains noncommutative free algebras over $k$. By what we have just seen, the subfields of $Q$ generated by these free algebras are not free fields.

We remark that the methods of G. Elek 8 provide an alternative proof for Corollary 5.3 in the case of algebras over the field of complex numbers.

\section{Free fields of uncountable Rank in Malcev-Neumann Series Rings}

This final section is devoted to the task of showing that Chiba's ideas in [3] can be explored further with the aim of showing that certain Malcev-Neumann series rings contain free fields of uncountable rank.

We start by recalling [3, Lemma 1], which will be used in the sequel.

Lemma 6.1. Let $D$ be a skew field with infinite centre and let $X$ be a set. Let $K$ be a subfield of $D$ which is its own bicentralizer and whose centralizer $K^{\prime}$ is such that the left $K$-space $K c K^{\prime}$ is infinite-dimensional over $K$ for all $c \in D^{\times}$. Let $L$ be a noncentral subnormal subgroup of the multiplicative group $K^{\prime \times}$, equivalently, $L$ is a subnormal subgroup of $K^{\prime \times}$ such that $L \nsubseteq K$. Then any full matrix over $D_{K}\langle X\rangle$ is invertible for some choice of values of $X$ in $L$.

The following result is a slightly more general version of [3, Lemma 2]. Note that we use multiplicative notation for the value group of $\nu$.

Lemma 6.2. Let $D$ be a skew field with a valuation $\nu$ on an ordered group $(G,<)$, and let $K$ be a subfield of $D$ which is its own bicentralizer and whose centralizer $K^{\prime}$ is such that the left $K$-space $K c K^{\prime}$ is infinite-dimensional over $K$, for all $c \in D^{\times}$. Let $L=\left\{x \in K^{\prime}: \nu(x)=1\right\}$. Then $L$ is a normal subgroup of the multiplicative group of $K^{\prime \times}$ and $L \nsubseteq K$.

Proof. For $c=1$, we get that $K K^{\prime}$ is infinite dimensional over $K$. Note also that the centre of $K^{\prime}$ is contained in $K$ since it is $K \cap K^{\prime}$, thus $K^{\prime}$ is infinite dimensional over its centre.

Suppose that $L \subseteq K$. Let $x \in K^{\prime} \backslash\left(K \cap K^{\prime}\right)$ with $\nu(x)>1$. Such an $x$ exists because $K^{\prime}$ is infinite-dimensional over $K \cap K^{\prime}$ and $L \subseteq K$. Observe that there exists $y \in K^{\prime}$ with $x y \neq y x$. Now $\nu(1-x)=\min \{\nu(1), \nu(x)\}=1$ but $y(1-x)=y-y x \neq y-x y=(1-x) y$. Thus $1-x \in L$, but $1-x \notin K, \mathrm{a}$ contradiction.

Let $(G,<)$ be an ordered group and let $\Gamma$ be the chain of its convex subgroups. Order $\Gamma$ with respect to inclusion, that is, given $H_{1}, H_{2} \in \Gamma$, set $H_{1} \prec H_{2}$ if and only if $H_{1} \subsetneq H_{2}$. The order type of $(\Gamma, \prec)$ is an invariant of $(G,<)$. Consider the subset $\Gamma_{0}$ of principal convex subgroups, that is, given $H \in \Gamma$, then $H \in \Gamma_{0}$ if there exists an element $t \in H$ such that $H=\left\{x \in G: t^{-n} \leq x \leq t^{n}\right.$, for some natural number $\left.n\right\}$. A convex subgroup $H$ is principal if and only if it is the greater member of a convex jump $(N, H)$ (see, for example, 9 ]).

Let $K$ be a skew field, let $(G,<)$ be an ordered group, let $K[G ; \sigma, \tau]$ be a crossed product group ring and let $K((G ; \sigma, \tau,<))$ be its Malcev-Neumann series ring. Now 
let $I$ be a set and suppose that there exists a map $I \rightarrow K((G ; \sigma, \tau,<)), i \mapsto f_{i}=$ $\sum_{x \in G} \bar{x} a_{i x}$, such that the following two conditions hold:

(1) $\bigcup_{i \in I} \operatorname{supp}\left(f_{i}\right)$ is well-ordered, and

(2) for each $x \in G$ the set $\left\{i \in I: x \in \operatorname{supp}\left(f_{i}\right)\right\}$ is finite.

Then, following [7], we say that $\sum_{i \in I} f_{i}$ is defined in $K((G ; \sigma, \tau,<))$. When this is the case, $\sum_{i \in I} f_{i}$ will be used to denote $\sum_{x \in G} \bar{x}\left(\sum_{\left\{i: x \in \operatorname{supp}\left(f_{i}\right)\right\}} a_{i x}\right)$. Note that $\sum_{i \in I} f_{i}$ is then an element of $K((G ; \sigma, \tau,<))$.

Lemma 6.3. Let $(G,<)$ be an ordered group, let $K$ be a skew field and let $K[G ; \sigma, \tau]$ be a crossed product. Let $\alpha$ be an ordinal. Suppose that the chain $\left(\Gamma_{0}, \prec\right)$ of principal convex subgroups of $(G,<)$ contains a subset $\left\{H_{\beta}\right\}_{\beta \leq \alpha}$ which is orderisomorphic to $\{\beta: \beta \leq \alpha\}$. For all $\beta \leq \alpha$, fix $1<t_{\beta}$ such that $H_{\beta}=\left\{x: t_{\beta}^{-n} \leq\right.$ $x \leq t_{\beta}^{n}$, for some natural number $\left.n\right\}$. If $\left\{f_{\beta}\right\}_{\beta \leq \alpha}$ is a sequence in $K((G ; \sigma, \tau,<))$ with supp $f_{\beta} \subseteq\left\{x \in H_{\beta}: x \geq 1\right\}$ for all $\beta \leq \alpha$, then $\sum_{\beta \leq \alpha} \bar{t}_{\beta} f_{\beta}$ is defined in $K((G ; \sigma, \tau,<))$.

Proof. First note that if $\beta_{1}<\beta_{2} \leq \alpha$, then $t_{\beta_{2}}^{-1}<t_{\beta_{1}}^{-n} \leq t_{\beta_{1}}^{n}<t_{\beta_{2}}$, for all natural numbers $n$, for otherwise $t_{\beta_{2}} \in H_{\beta_{1}}$ and thus $H_{\beta_{1}}=H_{\beta_{2}}$, a contradiction. Hence, since supp $f_{\beta} \subseteq\left\{x \in H_{\beta}: x \geq 1\right\}$ and $H_{\beta}=\left\{x \in G: t_{\beta}^{-n} \leq x \leq\right.$ $t_{\beta}^{n}$, for some natural number $\left.n\right\}$, for each $\beta \leq \alpha$, we get that $x_{1}<x_{2}$ for each $\beta_{1}<\beta_{2}$ and $x_{1} \in \operatorname{supp} \bar{t}_{\beta_{1}} f_{\beta_{1}}, x_{2} \in \operatorname{supp} \bar{t}_{\beta_{2}} f_{\beta_{2}}$. Therefore, for each $x \in G$, the set $\left\{\beta \leq \alpha: x \in \operatorname{supp} \bar{t}_{\beta} f_{\beta}\right\}$ is finite.

Now let $S$ be a nonempty subset of $\cup_{\beta \leq \alpha} \operatorname{supp} \bar{t}_{\beta} f_{\beta}$. Let $\beta_{0}=\min \{\beta: S \cap$ $\left.\operatorname{supp} \bar{t}_{\beta} f_{\beta} \neq \emptyset\right\}$. Then the least element of the well-ordered set $S \cap \operatorname{supp} \bar{t}_{\beta_{0}} f_{\beta_{0}}$ is the least element of $S$. Hence $\sum_{\beta \leq \alpha} \bar{t}_{\beta} f_{\beta}$ is defined in $K((G ; \sigma, \tau,<))$.

The proof of the next result is similar to that of [3, Theorem 1]. We recall the following fact that will be used in the proof. Let $D$ be a skew field with centre $C$. Let $X$ be a set and consider $D_{C}\langle X\rangle$. Let $a, t \in D$, with $t \neq 0$. Then there exists a unique isomorphism of $D$-rings $D_{C}\langle X\rangle \rightarrow D_{C}\langle X\rangle$ with $x \mapsto a+t x$, for all $x \in X$. Hence, if $A(x)$ is a full matrix over $D_{C}\langle X\rangle$, then $A(a+t x)$ is also a full matrix over $D_{C}\langle X\rangle$.

Theorem 6.4. Let $(G,<)$ be an ordered group, let $K$ be a skew field and let $K[G ; \sigma, \tau]$ be a crossed product. Let $\zeta$ be an infinite cardinal and let $X=\left\{x_{\gamma}\right\}_{\gamma<\zeta}$ be a set of cardinality $\zeta$. Suppose that the following two conditions hold true.

(a) The chain $\left(\Gamma_{0}, \prec\right)$ of principal convex subgroups of $(G,<)$ contains a subset $\left\{H_{\alpha}\right\}_{\alpha<\zeta}$ which is order-isomorphic to $\zeta$.

(b) For all $\alpha<\zeta$, the centre $C_{\alpha}$ of $E_{\alpha}=K\left(\left(H_{\alpha} ; \sigma, \tau,<\right)\right)$ is infinite and $\left[E_{\alpha}: C_{\alpha}\right]=\infty$.

Then $K((G ; \sigma, \tau,<))$ contains a free field $C(\langle X\rangle)$, where $C$ denotes the centre of $K((G ; \sigma, \tau,<))$.

Proof. Let $P$ be the prime subfield of $C$. Let $\Sigma$ be the set of all full matrices over $P\langle X\rangle$. Then $\Sigma$ is of cardinality $\zeta$. Write $\Sigma=\left\{A_{\alpha}\left(x_{\gamma}\right)\right\}_{\alpha<\zeta}$. For each $\alpha<\zeta$, fix $1<t_{\alpha} \in H_{\alpha}$ such that $H_{\alpha}=\left\{x \in G: t_{\alpha}^{-n} \leq x \leq t_{\alpha}^{n}\right.$, for some natural number $\left.n\right\}$.

By [3, Lemma 9], it is enough to prove that $P(\langle X\rangle)$ embeds into $E=K((G ; \sigma, \tau,<$ ) ). We will show that there exists an honest embedding $P\langle X\rangle \hookrightarrow E$, that is, there 
exists $\left\{d_{\gamma}\right\}_{\gamma<\zeta} \subseteq E$ such that the matrix $A_{\alpha}\left(d_{\gamma}\right)$ is invertible for all $\alpha<\zeta$. The first part of the proof is devoted to the construction of these $d_{\gamma}, \gamma<\zeta$.

Let $\omega: E \rightarrow G_{\infty}$ denote the natural valuation given by $\omega(f)=\min \operatorname{supp} f$, for all nonzero $f \in E$. We define sequences $\left\{h_{\gamma \beta}: \gamma, \beta<\zeta\right\}$ satisfying the following conditions.

(i) $h_{\gamma \alpha} \in V_{\alpha}^{\prime}=\left\{f \in E_{\alpha}: \omega(f) \geq 1\right\}$, for all $\alpha<\zeta$.

(ii) For each $\beta, h_{\gamma \beta}=0$ for almost all but a finite number of $\gamma$.

(iii) $\sum_{\beta \leq \alpha} \bar{t}_{\beta} h_{\gamma \beta}$ is defined in $E_{\alpha}$.

(iv) $A_{\alpha}\left(\sum_{\beta \leq \alpha} \bar{t}_{\beta} h_{\gamma \beta}\right)$ is invertible over $E_{\alpha}$, for all $\alpha<\zeta$.

First, observe that (iii) always holds by Lemma 6.3.

Set $L_{0}=\left\{x \in E_{0}: \omega(x)=1\right\}$. Then $L_{0} \nsubseteq C_{0}$, by Lemma 6.2, Consider the finite set of $x$ 's which appear in the matrix $A_{0}\left(x_{\gamma}\right)$, e.g. $x_{0}, x_{1}, \ldots, x_{r}$. Since $A_{0}\left(\bar{t}_{0} x_{\gamma}\right)$ is a full matrix over $E_{0 C_{0}}\langle X\rangle$, there are elements $h_{\gamma 0} \in L_{0}, \gamma=1, \ldots, r$, such that $A_{\gamma}\left(\bar{t}_{0} h_{\gamma 0}\right)$ is an invertible matrix over $E_{0}$, by Lemma 6.1 Set $h_{\gamma 0}=0$ for $\gamma \notin\{1, \ldots, n\}$. Let $B_{0}$ be the inverse of $A_{0}\left(\bar{t}_{0} h_{\gamma 0}\right)$. There exists a natural number $b_{0}>0$ such that $B_{0}=\bar{t}_{0}^{-b_{0}} B_{0}^{0}$, where all entries of $B_{0}^{0}$ are elements of $V_{01}=\left\{f \in E_{0}: \omega(f) \geq 1\right\}$.

Let $0<\alpha<\zeta$. Suppose that we have defined $h_{\gamma \beta}$ for $\gamma<\zeta, \beta<\alpha$ satisfying (ii)-(iv). Set $L_{\alpha}=\left\{f \in E_{\alpha}: \omega(f)=1\right\}$. Then $L_{\alpha} \nsubseteq \nsubseteq C_{\alpha}$, by Lemma 6.2 Consider the finite set $\left\{x_{\gamma_{1}}, \ldots, x_{\gamma_{s}}\right\} \subseteq X$ which appear in the matrix $A_{\alpha}\left(x_{\gamma}\right)$. Since $A_{\alpha}\left(\sum_{\beta<\alpha} \bar{t}_{\beta} h_{\gamma \beta}+\bar{t}_{\alpha} x_{\alpha}\right)$ is a full matrix over $E_{\alpha C_{\alpha}}\langle X\rangle$, there are $h_{\gamma \alpha} \in L_{\alpha}$, $\gamma=\gamma_{1}, \ldots, \gamma_{s}$, such that $A_{\alpha}\left(\sum_{\beta \leq \alpha} \bar{t}_{\beta} h_{\gamma \beta}\right)$ is an invertible matrix over $E_{\alpha}$, by Lemma 6.1] Set $h_{\gamma \alpha}=0$ for $\gamma \notin\left\{\gamma_{1}, \ldots, \gamma_{s}\right\}$. Let $B_{\alpha}$ be the inverse matrix of $A_{\alpha}\left(\sum_{\beta \leq \alpha} \bar{t}_{\beta} h_{\gamma \beta}\right)$. There exists a natural number $b_{\alpha}>0$ such that $B_{\alpha}=\bar{t}_{\alpha}^{-b_{\alpha}} B_{\alpha}^{0}$, where all the entries of $B_{\alpha}^{0}$ are elements $V_{\alpha 1}=\left\{f \in E_{\alpha}: \omega(f) \geq 1\right\}$.

Now it is easy to see that $d_{\gamma}=\sum_{\alpha<\zeta} \bar{t}_{\alpha} h_{\gamma \alpha}$ is defined in $E$, for each $\gamma<\zeta$.

We now show that $A_{\alpha}\left(d_{\gamma}\right)=A_{\alpha}\left(\sum_{\alpha<\zeta} \bar{t}_{\alpha} h_{\gamma \alpha}\right)$ is an invertible matrix over $E$, for all $\alpha<\zeta$. For each $\alpha<\zeta$, we have

$$
B_{\alpha}^{0} \cdot A_{\alpha}\left(\sum_{\beta \leq \alpha} \bar{t}_{\beta} h_{\gamma \beta}\right)=\left(\begin{array}{ccc}
\bar{t}_{\alpha}^{b_{\alpha}} & & 0 \\
& \ddots & \\
0 & & \bar{t}_{\alpha}^{b_{\alpha}}
\end{array}\right)
$$

and

$$
\sum_{\beta<\zeta} \bar{t}_{\beta} h_{\gamma \beta} \equiv \sum_{\beta \leq \alpha} \bar{t}_{\beta} h_{\gamma \beta} \quad \bmod \left(V_{t_{\alpha+1}}\right),
$$

where $V_{t_{\alpha+1}}=\left\{f \in E: \omega(f) \geq t_{\alpha+1}\right\}$. Hence,

$$
B_{\alpha}^{0} \cdot A_{\alpha}\left(\sum_{\beta<\zeta} \bar{t}_{\beta} h_{\gamma \beta}\right)=\left(\begin{array}{ccc}
\bar{t}_{\alpha}^{b_{\alpha}} & & 0 \\
& \ddots & \\
0 & & \bar{t}_{\alpha}^{b_{\alpha}}
\end{array}\right)+M^{\prime},
$$

where the entries of $M^{\prime}$ are in $V_{t_{\alpha+1}}$. Since $\left\{\bar{t}_{\alpha}^{b_{\alpha}}(1-M)\right\}^{-1}=\left(1+M+M^{2}+\cdots\right) \bar{t}^{-b_{\alpha}}$, where $M$ is a matrix over $V_{1}=\{f \in E: \omega(f)>1\}$, it follows that $A_{\alpha}\left(d_{\gamma}\right)$ is invertible over $E$.

In the proof of the foregoing result, we have used each $t_{\beta}$ to invert a matrix of $\Sigma$. We remark that in [3. Theorem 1], with only one $t$, an infinite countable number of matrices are inverted. We could have done something similar, but it would not be 
of any help because the set of $t$ 's has to be of cardinality $\zeta$ since $X$ is of uncountable cardinality $\zeta$.

Our next goal is to prove a result similar to Corollary 2.4 for an uncountable set $X$. For that we make a free abelian group into an ordered group with a chain of principal convex subgroups which is order-isomorphic to a certain cardinal.

Let $\zeta$ be a cardinal, and let $G_{\zeta}$ be the free abelian group with a basis $\left\{e_{\beta}: \beta<\zeta\right\}$. Thus every nonzero element $x$ of $G_{\zeta}$ is uniquely expressed as

$$
x=m_{1} e_{\beta_{1}}+\cdots+m_{r} e_{\beta_{r}},
$$

with $m_{i} \in \mathbb{Z} \backslash\{0\}$ and ordinals $\beta_{1}<\cdots<\beta_{r}<\zeta$.

We now make $G_{\zeta}$ into an ordered group in the following way. Given $x$ as in (1), we say that $x>0$ if and only if $m_{r}>0$. Then given $x, y \in G_{\zeta}$, it is not difficult to see that the relation given by $x>y$ whenever $x-y>0$ is a total order in $G_{\zeta}$ and that $\left(G_{\zeta},<\right)$ is an ordered group.

For each ordinal $\alpha<\zeta$, let $H_{\alpha}$ be the subgroup generated by $\left\{e_{\beta}\right\}_{\beta \leq \alpha}$. Note that $H_{\alpha}$ is a convex subgroup of $\left(G_{\zeta},<\right)$. In fact, $H_{\alpha}$ is a principal convex subgroup of $\left(G_{\zeta},<\right)$, because $H_{\alpha}=\left\{x \in G:-n e_{\alpha} \leq x \leq n e_{\alpha}\right.$, for some natural number $\left.n\right\}$.

Corollary 6.5. Let $K$ be a skew field with centre $F$ such that the dimension of $K$ over $F$ is infinite. Let $X$ be a set of cardinality $\zeta$. Consider the ordered group $\left(G_{\zeta},<\right)$. Then the field $K\left(\left(G_{\zeta} ;<\right)\right)$ contains a free field $F(\langle X\rangle)$.

Proof. We show that the conditions (a) and (b) of Theorem 6.4 are satisfied. The chain of principal convex subgroups $\left\{H_{\alpha}: \alpha<\zeta\right\}$ of $G_{\zeta}$ is isomorphic, as an ordered set, to the cardinal $\zeta$. For each $\alpha<\zeta$, it can be proved that a series $f \in E_{\alpha}=$ $K\left(\left(H_{\alpha} ;<\right)\right)$ commutes with each $d \in K$ if and only if $f \in F_{\alpha}=F\left(\left(H_{\alpha} ;<\right)\right)$. Thus $F_{\alpha}$ is the centre of $E_{\alpha}$. Similarly, the centre of $K\left(\left(G_{\zeta} ;<\right)\right)$ is $F\left(\left(G_{\zeta} ;<\right)\right)$. Now if $\left\{d_{i}\right\}_{i \in I}$ is a basis of $K$ as an $F$-vector space, then the set $\left\{d_{i}\right\}_{i \in I}$ is a subset of $E_{\alpha}$ which is linear independent over $F_{\alpha}$. Hence the dimension $\left[E_{\alpha}: F_{\alpha}\right]$ is infinite. It follows from Theorem 6.4 that $K\left(\left(G_{\zeta} ;<\right)\right)$ contains a free field $F\left(\left(G_{\zeta} ;<\right)\right)(\langle X\rangle)$. In particular, it contains $F(\langle X\rangle)$.

\section{REFERENCES}

[1] V. V. Bavula, Finite generation of the group of eigenvalues for sets of derivations or automorphisms of division algebras, Comm. Algebra 36 (2008), 2195-2201.

[2] R. Botto Mura and A. Rhemtulla, Orderable groups, Marcel Dekker, New York, 1977.

[3] K. ChiBA, Free fields in complete skew fields and their valuations, J. Algebra 263 (2003), $75-87$.

[4] P. M. Conn, Skew Field Constructions, Cambridge University Press, Cambridge, 1977.

[5] P. M. Cohn, Free Rings and Their Relations, 2nd. Ed., Academic Press, London, 1985.

[6] P. M. Conn, Skew Fields. Theory of General Division Rings, Cambridge University Press, Cambridge, 1995.

[7] W. Dicks, And J. Lewin, A Jacobian conjecture for free associative algebras, Comm. Algebra 10 (1982), 1285-1306.

[8] G. Elek, The amenability and non-amenability of skew fields, Proc. Amer. Math. Soc. 134 (2006), 637-644.

[9] L. Fuchs, Partially Ordered Algebraic Systems, Pergamon Press, Oxford, 1963.

[10] J. Z. Gonçalves and M. Shirvani, A survey on free objects in division rings and in division rings with an involution, to appear in Comm. Algebra.

[11] I. Hughes, Division rings of fractions for group rings, Comm. Pure Appl. Math. 23 (1970), $181-188$.

[12] S. A. Jennings, The group ring of a class of infinite nilpotent groups, Canad. J. Math. 7 (1955), 169-187. 
[13] T. Y. Lam, A First Course in Noncommutative Rings, 2nd. Ed., Springer-Verlag, New York, 2001.

[14] T. Y. LAM, Lectures on Modules and Rings, Springer-Verlag, New York, 1999.

[15] J. Lewin, Fields of fractions for group algebras of free groups, Trans. Amer. Math. Soc. 192 (1974), 339-346.

[16] A. I. Lichtman, On subgroups of the multiplicative group of skew fields, Proc. Amer. Math. Soc. 63 (1977), 15-16.

[17] A. I. Lichtman, Valuation methods in division rings, J. Algebra 177 (1995), 870-898.

[18] A. I. Lichtman, Free subalgebras in division rings generated by universal enveloping algebras, Algebra Colloq. 6 (1999), 145-153.

[19] L. Makar-Limanov, The skew field of fractions of the Weyl algebra contains a free noncommutative subalgebra, Comm. Alg. 11 (1983), 2003-2006.

[20] L. Makar-Limanov, On group rings of nilpotent groups, Israel J. Math. 48 (1984), 244-248.

[21] L. Makar-Limanov and P. Malcolmson, Free subalgebras of enveloping fields, Proc. Amer. Math. Soc. 111 (1991), 315-322.

[22] A. I. Malcev, On the embedding of group algebras in division algebras, Doklady Akad. Nauk SSSR (N.S.) 60 (1948), 1499-1501.

[23] A. Navas and C. Rivas, Describing all bi-orderings on Thompson's group F, Groups Geom. Dyn. 4 (2010), 163-177.

[24] B. H. Neumann, On ordered division rings, Trans. Amer. Math. Soc. 66 (1949), 202-252.

[25] D. S. Passmann, Infinite Crossed Products, Academic Press, Boston, MA, 1989.

[26] M. E. SweEdLer, Tensor products of division rings and finite generation of subdivision rings, Bull. Inst. Math. Acad. Sinica 8 (1980), 385-387.

Department of Mathematics - Ime, University of São Paulo, Caixa Postal 66281, São PAUlo, SP, 05314-970, BRAZIL

E-mail address: vofer@ime.usp.br

Department of Mathematics, State University of Maringá, Avenida Colombo, 5790, MARINGÁ, PR, 87020-900, BRAZIL

E-mail address: ezancanella@uem.br

Department of Mathematics - Ime, University of São Paulo, Caixa Postal 66281, São PAUlO, SP, 05314-970, BRAZIL

E-mail address: jsanchez@ime.usp.br 